\title{
On the prospect of bioelectronics and biosensors with the novel topological phases of matter
}

\begin{abstract}
Herein, the application capabilities of novel topological phases of matter in the field of bioelectronics and biosensors are assessed. Topological insulators could enable a novel generation of biosensors, exploiting the reduction of the low-frequency noise arising from the suppressed backscattering. Case-study examples of biosensors based on bismuth chalcogenides are discussed.
\end{abstract}

Keywords: topological insulators, biosensors, bioelectronics, low-frequency noise

\author{
Volume 2 Issue 4 - 2017
}

\author{
Antonio Politano \\ Dipartimento di Fisica, Università della Calabria, Italy
}

Correspondence: Antonio Politano, Dipartimento di Fisica, Università della Calabria, via ponte Bucci, cubo 3I/C, Rende (CS), Tel +39-0984-496107, Fax +39-0984-49440I, Email antonio.politano@fis.unical.it

Received: April 19, 2017 | Published: April 24, 2017

\section{Opinion}

Biosensors are devices of great importance for medical diagnostics, therapy monitoring, environmental science, biotechnology etc. The recent achievements in this fields are based on research on (i) materials and (ii) optimization of processes. By adopting standard solutions, further noteworthy advancement is unfeasible. Conversely, unexplored materials could provide interesting perspectives. The isolation of graphene in 2004 has paved the way for disruptive technologies based on two-dimensional materials. ${ }^{1,2}$ However, the absence of a band gap in graphene ${ }^{3}$ impedes the achievement of a good ON/OFF ratio in nanodevices. ${ }^{4}$ Following graphene, other twodimensional van der Waals semiconductors, combining finite band gaps $^{5,6}$ and flexibility ${ }^{7,8}$ are emerging in recent years as the most promising materials for nanoelectronics. ${ }^{49}$ Topological insulators (TIs) are a class of innovative materials, whose physical properties have enormous potential for technological applications. ${ }^{10}$

TIs represent unique phases of matter with semiconducting bulk and conducting edge or surface states, immune to small perturbations, such as backscattering due to disorder. This stems from their peculiar band structure, which provides topological protection. Moreover, electron spin and momentum in topologically protected states are locked, thus enabling (i) the control of electron flow and (ii) the reduction of low-frequency noise in TI-based electronic devices. ${ }^{11}$ As a matter of fact, the suppression of the backscattering of spin-polarized charge carriers of surface states, with the subsequent suppression of the fluctuation of mobility of surface-state charge carriers, could reduce electronic noise, so as to lower the detection limits of sensors below those already reported. ${ }^{11}$

Despite great interest from the scientific community, the huge potential of TIs for technological applications has been just started to be exploited. ${ }^{12}$ Recent works report superb performance in TI-based nanodevices, ${ }^{13}$ also thanks to the progress in the control of singlecrystal quality during the TI growth process. Among TIs, layered bismuth chalcogenides have attracted much attention since they can be easily grown by Bridgman-Stockbarger method and, moreover, since they show TI properties at room temperature. ${ }^{13}$

The discovery of the unique properties of TIs, such as fast electron transportation, ${ }^{14}$ high thermal conductivity, ${ }^{15}$ good biocompatibility, ${ }^{16}$ can give rise to a new generation of biosensors based on TIs. Glucose sensors based on $\mathrm{Bi} 2 \mathrm{Se} 3$ have been developed by exploiting the high electrochemical catalytic activity of $\mathrm{Bi} 2 \mathrm{Se} 3$ for the reduction of dissolved $\mathrm{O}_{2}{ }^{17}$ Likewise, prussian blue/Bi2Se3 hybrid films with immobilized glucose oxidase have been used to fabricate an amperometric glucose biosensor. ${ }^{18}$ The detection limit was estimated for $3.8 \mu \mathrm{M}$ defined from a signal/noise of 3 . The resulting biosensor was tested to detect the blood sugar in human serum samples. ${ }^{18}$

Recently, an effective colorimetric biosensor based on highly catalytic active $\mathrm{Au}$ nanoparticle-decorated $\mathrm{Bi} 2 \mathrm{Se} 3$ has been fabricated. ${ }^{19}$ The low redox potential of Bi2Se3 and its TI properties enable providing and accumulating electrons at the surface. Such unique properties contribute to strong synergistic catalytic effects with gold nanoparticles. The outstanding catalytic activity of $\mathrm{Au} /$ Bi2Se3 nanosheets can be "switched off" upon treatment of antibody of cancer biomarker such as anticarcinoembryonic antibody (antiCEA). Adding the corresponding antigen such as cancer biomarker carcinoembryonic antibody (CEA) can sequentially favour "switch on" the catalytic activity of $\mathrm{Au} / \mathrm{Bi} 2 \mathrm{Se} 3$. The system can be thus used as a colorimetric sensor for detection of a particular cancer biomarker with high sensitivity and selectivity for the cancer biomarker, even for a concentration as low as $160 \mathrm{pg} / \mathrm{mL}$ for CEA. The devised colorimetric sensor shows good generality for detecting different kinds of cancer biomarkers, such as $\alpha$-fetoprotein (AFP) and prostate-specific antigen (PSA). Moreover, the devised biosensor is efficient for detection of CEA, thus providing an alternative technique in cancer diagnosis.

\section{Conclusion}

In conclusion, bioelectronics and biosensors based on TIs are just at their infancy, in spite of their high potential. However, the creation of new disruptive technologies based on TIs is conditional to reaching a variety of objectives and overcoming several challenges

\section{Acknowledgements}

None.

\section{Conflict of interest}

The author declares no conflict of interest. 


\section{References}

1. Ferrari AC, Bonaccorso F, Falko V, et al. Science and technology roadmap for graphene, related two-dimensional crystals, and hybrid systems. Nanoscale. 2015;7(11):4598-4810

2. Cupolillo, Anna, Ligato, et al. Low energy two-dimensional plasmon in epitaxial graphene on Ni (111). Surf Sci. 2013;608:88-91.

3. Novoselov K. Graphene: Mind the gap. Nat Mater. 2007;6(10):720-721.

4. Fiori G, Bonaccorso F, Iannaccone G, et al. Electronics based on twodimensional materials. Nat Nanotech. 2014;9(10):768-779.

5. Miro P, Audiffred M, Heine T. An atlas of two-dimensional materials. Chem Soc Rev. 2014;43(18):6537-6554.

6. Bhimanapati GR, Lin Z, Meunier V, et al. Recent advances in twodimensional materials beyond graphene. ACS Nano. 2015;9(12):1150911539.

7. Akinwande D, Petrone N, Hone J. Two-dimensional flexible nanoelectronics. Nat Commun. 2014;5:5678.

8. Zhu W, Yogeesh MN, Yang S, et al. Flexible black phosphorus ambipolar transistors, circuits and am demodulator. Nano Lett. 2015;15(3):18831890.

9. Koppens FH, Mueller T, Avouris P, et al. Photodetectors based on graphene, other two-dimensional materials and hybrid systems. Nat Nanotechnol. 2014;9(10):780-793.

10. Hasan MZ, CL Kane. Colloquium: Topological insulators. Rev Mod Phys. 2010;82(4):3045-3067.
11. Hossain, Rumyantsev MZSL, Teweldebrhan D, et al. 1/f noise in conducting channels of topological insulator materials. Phys Status Solidi A. 2011;208(1):144-146.

12. Politano A, Viti L, Vitiello MS. Optoelectronic devices, plasmonics and photonics with topological insulators. APL Materials. 2017;5:035504.

13. Viti L, Coquillat D, Politano A, et al. Plasma-wave terahertz detection mediated by topological insulators surface states. Nano Lett. 2016;16(1):80-87.

14. Taskin AA, Sasaki S, Segawa K, et al. Manifestation of topological protection in transport properties of epitaxial Bi2Se3 thin films. Phys Rev Lett. 2012;109(6):066803.

15. Guozhi J, Peng W, Yanbang Z, et al. Localized surface plasmon enhanced photothermal conversion in Bi2Se3 topological insulator nanoflowers. Sci Rep. 2016;6:25884.

16. Zhang XD. Metabolizable Bi2Se3 Nanoplates: biodistribution, toxicity, and uses for cancer radiation therapy and imaging. Adv Funct Mater. 2014;24(12):1718-1729.

17. Chen S, YM Fang, J Li, et al. Study on the electrochemical catalytic properties of the topological insulator Bi2Se3. Biosensors and Bioelectronics. 2013;46:171-174.

18. Wu S, Liu G, Li P, et al. A high-sensitive and fast-fabricated glucose biosensor based on Prussian blue/topological insulator Bi2Se3 hybrid film. Biosens Bioelectron. 2012;38(1):289-294.

19. Xiao L, Zhu A, Xu Q, et al. Colorimetric biosensor for detection of cancer biomarker by au nanoparticle-decorated Bi2Se3 nanosheets. ACS Appl Mater Interfaces. 2017;9(8):6931-6940. 